Pacific Journal of Mathematics

ON COVERINGS OF FIGURE EIGHT KNOT SURGERIES 


\title{
ON COVERINGS OF FIGURE EIGHT KNOT SURGERIES
}

\author{
MARK D. BAKER
}

\begin{abstract}
We show that over half of the Dehn surgeries on $S^{3}$ along the figure eight knot $K$ yield manifolds having finite covers with positive first Betti number by explicitly constructing these covers and exhibiting their homology.
\end{abstract}

1. Introduction. Denote by $K$ the figure eight knot, pictured in Figure 1. In his celebrated Notes, $[\mathrm{T}]$, Thurston showed that all but finitely many Dehn surgeries along $K$ in $S^{3}$ yield hyperbolic nonHaken manifolds - the first such examples. It remains an open question whether or not these manifolds (or every closed, irreducible 3manifold with infinite $\pi_{1}$ ) are finitely covered by Haken manifolds, or stronger still, by manifolds with positive first Betti number.

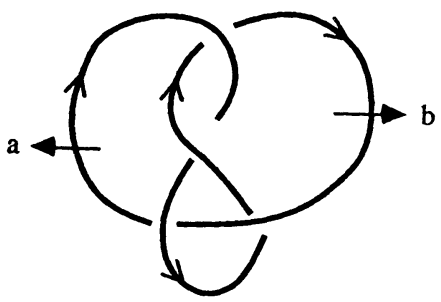

FIGURE 1

In this paper we will show that over half of the Dehn surgeries along $K$ yield manifolds having finite covers with positive first Betti number by explicitly constructing these covers and exhibiting their homology.

Section 2 is devoted to notation and preliminaries. Section 3 contains a statement of our results as well as a summary of previous results on the problem. The method of proof is outlined in $\S 4$. Proofs are given in $\S \S 5-7$.

2. Preliminaries. Throughout this paper $K$ will denote the figure eight knot and $M$ the complement, in $S^{3}$, of an open regular neighborhood of $K$. We will use the fact that $M$ is a bundle over $S^{1}$ with fiber a once-punctured torus.

2.1. Let $T_{0}$ denote the torus with an open disk removed, pictured in Figure 2. Let $D_{x}$ denote the left-handed Dehn twist about the loop 


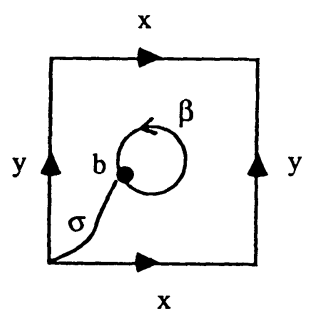

FIGURE 2

$x$ and $D_{y}$ the right-handed Dehn twist about the loop $y$ in $T_{0}$. Then

$$
M \cong T_{0} \times[0,1] /(g(s), 0) \sim(s, 1)
$$

where $g=D_{x} \circ D_{y}$.

We fix a basepoint, $b$, in $\partial T_{0}$ and let $x, y$ be the elements of $\pi_{1}\left(T_{0}, b\right)$ represented by the loops $x, y$ in $T_{0}$ based at $b$ via the arc $\sigma$. Then $x$ and $y$ freely generate $\pi_{1}\left(T_{0}, b\right)$ and $D_{x}, D_{y}$ induce the isomorphisms:

$$
\begin{aligned}
& \left(D_{x}\right)_{\#}: x \rightarrow x, \quad y \rightarrow y x, \\
& \left(D_{y}\right)_{\#}: x \rightarrow x y, \quad y \rightarrow y .
\end{aligned}
$$

The loop $\alpha=b \times[0,1] / \sim$ is a meridian for $K$ and $\beta=\partial T_{0}$ is a longitude for $K$. Then

$$
\pi_{1}(M) \cong\left\langle x, y, \alpha \mid \alpha^{-1} x \alpha=x y x, \alpha^{-1} y \alpha=y x\right\rangle
$$

which is easily seen to be isomorphic to the following Wirtinger presentation for $S^{3} \backslash K$ :

$$
\pi_{1}\left(S^{3} \backslash K\right) \cong\left\langle a, b \mid\left(a^{-1} b a b^{-1}\right) a\left(a^{-1} b a b^{-1}\right)^{-1} b^{-1}=\mathrm{id}\right\rangle .
$$

Indeed, first eliminate $y \quad\left(y=x^{-1} \alpha^{-1} x \alpha x^{-1}\right)$ then set $\alpha=a^{-1}$ and $x=b a^{-1}$.

2.2. By Dehn filling on a 3-manifold $X$ with respect to a loop in a boundary torus, we mean attaching a solid torus to $\partial X$ so that this loop bounds a meridional disk in the solid torus.

We say that $X$ has a virtually $\mathbb{Z}$-representable fundamental group if $\pi_{1}(X)$ contains a finite index subgroup with non-trivial representation to $\mathbb{Z}$. If $X$ is compact, this is equivalent to the existence of a finite cover $\widetilde{X} \rightarrow X$ with $\beta_{1}(\widetilde{X}) \equiv \operatorname{rank} H_{1}(\widetilde{X})>0$.

Given a surface $F$ and a homeomorphism $h: F \rightarrow F$, we define the corresponding bundle over $S^{1}$ by $F \times I / h \equiv F \times[0,1] /(h(s), 0) \sim$ $(s, 1)$. Note that the back face $F \times\{1\}$ is attached to the front face $F \times\{0\}$ via $h$. 
Given $M_{h}=T_{0} \times I / h$ with $h$ the identity on $\partial T_{0}$, define (as for $M)$ the loops $\alpha_{h}=b \times I / \sim, \beta=\partial T_{0}$.

Definitions. (1) $M_{h}(\mu, \lambda)$ represents the manifold obtained by Dehn filling on $M_{h}$ with respect to the loop $\alpha_{h}^{\mu} \beta^{\lambda}$.

(2) By $\mu / \lambda$ Dehn surgery along $K$ in $S^{3}$, we mean Dehn filling on $M$ with respect to $\alpha^{\mu} \beta^{\lambda}$. Let $M(\mu, \lambda)$ denote the resulting manifold.

REMARKs. (1) $M(\mu, \lambda) \cong M(\mu,-\lambda)$ since there exists an orientation reversing homeomorphism on $M$ sending $\alpha$ to $\alpha$ and $\beta$ to $\beta^{-1}$ (see [H2] or [T]).

(2) Since $M_{h}(\mu, \lambda)=M_{h}(-\mu,-\lambda)$ we will assume that $\mu \geq 1$.

3. Statement of results. $M(\mu, \lambda)$ is known to have a virtually $\mathbb{Z}$ representable fundamental group if:

(i) $\lambda \equiv \pm 2 \mu(\bmod 7) \quad($ see $[\mathrm{H1}]$ or $[\mathbf{N}])$,

(ii) $\lambda \equiv \pm \mu(\bmod 13) \quad($ see $[\mathrm{H1}])$,

(iii) $\mu \equiv 0(\bmod 4)$ and $\mu / \lambda \neq \pm 8 \quad($ see $[K L])$.

In $\S 5$ below, we will prove:

THEOREM A. $M(3 \mu, \lambda)$ has a virtually $\mathbb{Z}$-representable fundamental group if $|\lambda| \notin\{\mu-1, \mu+1\}$.

In $\S 6$, we first give a simple proof of (iii) by explicitly constructing covers $N \rightarrow M(4 \mu, \lambda)$, for which $\beta_{1}(N) \geq 1$. We show that $M(8, \pm 1)$ has a virtually $\mathbb{Z}$-representable fundamental group, the case not covered in $[\mathbf{K L}]$. We then prove virtual $\mathbb{Z}$-representablility for certain $M(2 \mu, \lambda)$ :

Proposition C. $M(2 \mu, \lambda)$ has a virtually $\mathbb{Z}$-representable fundamental group if $\lambda \equiv \pm 7 \mu(\bmod 15)$.

In $\S 7$, we study singular boundary curve systems for $M$. In [H2], it is shown that $\left\{\alpha^{3}\right\},\{\alpha \beta\}$ and $\left\{\alpha \beta^{-1}\right\}$ are singular boundary curve systems. We prove the following result:

THEOREM D. $\left\{\alpha^{2} \beta\right\},\left\{\alpha^{2} \beta^{-1}\right\},\left\{\alpha^{3} \beta\right\}$ and $\left\{\alpha^{3} \beta^{-1}\right\}$ are singular boundary curve systems for $M$.

REMARK. Our results, combined with (i)-(iii) above, show that approximately two-thirds of the surgeries on $K$ yield manifolds having virtually $\mathbb{Z}$-representable fundamental groups. 
4. Construction of covers. For a given $(\mu, \lambda)$, we show that $M(\mu, \lambda)$ has a virtually $\mathbb{Z}$-representable fundamental group by constructing a finite cover $N \rightarrow M(\mu, \lambda)$ with $\beta_{1}(N) \equiv \operatorname{rank} H_{1}(N) \geq 1$. The cover $N$ is obtained from a finite cover $\widetilde{M} \rightarrow M$ having the following two properties:

(i) The loop $\alpha^{\mu} \beta^{\lambda}$ in $\partial M$ lifts to loops in the components of ә $\widetilde{M}$

(ii) $\beta_{1}(\widetilde{M})>\beta_{0}(\partial \widetilde{M})$.

Property (i) guarantees that $\widetilde{M} \rightarrow M$ extends to an (unbranched) cover $N \rightarrow M(\mu, \lambda)$ by Dehn filling on $\widetilde{M}$ and $M$. Property (ii) guarantees that any manifold obtained by Dehn filling on $\widetilde{M}$ (hence $N)$ has positive first Betti number.

Since $M$ is a bundle over $S^{1}$ with fiber $T_{0}$ and characteristic homeomorphism $g$, it follows that $\widetilde{M}$ is also a bundle over $S^{1}$ with fiber $F$ a cover of $T_{0}$ and characteristic homeomorphism $\tilde{g}$ a lifting of $g^{n}$ for some integer $n \geq 1$.

It is easy to show (see [H1]) that $\widetilde{M}$ satisfies property (ii) above if and only if $\tilde{g}_{*}: H_{1}(F) \rightarrow H_{1}(F)$ fixes a non-boundary class in $H_{1}(F)$. We adopt the terminology of [H1] that $\tilde{g}$ is homology reducible if it fixes such a non-boundary class in $H_{1}(F)$.

Thus we will construct $\widetilde{M}$ by constructing a finite cover $F \rightarrow T_{0}$ to which an appropriate power of $g$ lifts to a homeomorphism $\tilde{g}: F \rightarrow$ $F$ that is homology reducible.

Since $g=D_{x} \circ D_{y}$, it is difficult to tell, given a cover $F \rightarrow T_{0}$, whether or not $g^{n}$ lifts to a $\tilde{g}$ that is homology reducible (in fact the matter of whether or not a given $g^{n}$ even lifts is difficult to verify in practice). We will avoid these difficulties by using the fact that $g^{2}$, $g^{3}$ and $g^{4}$ are isotopic to maps that are much easier to work with.

5. In this section we prove the following:

THEOREM A. $M(3 \mu, \lambda)$ has a virtually $\mathbb{Z}$-representable fundamental group if $|\lambda| \notin\{\mu-1, \mu+1\}$.

We fix $h=D_{x}^{2} \circ D_{y}^{-4} \circ D_{x} \circ D_{y}^{-4} \circ D_{x}$. Recall that $M_{h}=T_{0} \times I / h$, $\alpha_{h}=b \times I / h, \beta=\partial T_{0}$ and $M_{h}(\mu, \lambda)$ is the manifold obtained by: Dehn filling on $M_{h}$ with respect to the loop $\alpha_{h}^{\mu} \beta^{\lambda}$ (see $\S 2.2$ ).

LEMMA 5.1. $M_{h}(\mu, \lambda) \rightarrow M(3 \mu, \mu+\lambda) \cong M(3 \mu,-\mu-\lambda)$ is a 3-fold cover. 




FIGURE 3

Proof. Since $h$ and $g^{3}$ both have the same monodromy matrix $\left(\begin{array}{cc}13 & 8 \\ 8 & 5\end{array}\right) \in \mathrm{SL}_{2}(\mathbb{Z})$ they are isotopic, and hence $M_{h}$ is bundle equivalent to the 3-fold cyclic cover, $M_{g^{3}}$, of $M$. Moreover the isotopy $H$ from $g^{3}$ to $h$ rotates $\partial T_{0}$ one turn counter-clockwise, since for any $z \in \pi_{1}\left(T_{0}, b\right)$,

$$
g_{\#}^{3}(z)=\left(x y x^{-1} y^{-1}\right) h_{\#}(z)\left(x y x^{-1} y^{-1}\right)^{-1} .
$$

(It suffices to check this for $x, y \in \pi_{1}\left(T_{0}, b\right)$.) Thus the induced bundle isomorphism $H: M_{h} \rightarrow M_{g^{3}}$ sends the pair of loops $\left(\alpha_{h}, \beta\right)$ to $\left(\alpha_{g^{3}} \beta, \beta\right)$ which projects to $\left(\alpha^{3} \beta, \beta\right)$ in $M$.

Now Theorem 1 of [B] tells us that $M_{h}(\mu, \lambda)$ has a virtually $\mathbb{Z}$ representable fundamental group for $\mu \geq 1,|\lambda| \geq 2$ and, if $\lambda$ is odd, either $\lambda>2$ or $-4 \mu / 3<\lambda<-2$ or $\lambda<-4 \mu$. Since $M_{h}(\mu, \lambda) \rightarrow$ $M(3 \mu, \mu+\lambda) \cong M(3 \mu,-\mu-\lambda)$ is a cover, Theorem A above follows easily.

5.2. We illustrate Theorem A by constructing covers $N \rightarrow M(3 \mu, \lambda)$, $\beta_{1}(N) \geq 1$, for $\mu, \lambda$ odd. Consider the 16-fold cover $F \rightarrow T_{0}$ pictured in Figure 3. Let $F^{\prime} \rightarrow T_{0}$ be the cover corresponding to the kernel of the map $\theta: \pi_{1}\left(T_{0}\right) \rightarrow \mathbb{Z} / 4 \oplus \mathbb{Z} / 4$ defined by $\theta([x])=(1,0)$ and $\theta([y])=(0,1)$. We obtain $F$ by making eight vertical cuts in $F^{\prime}$ and identifying the left edge of each cut to the right edge of the cut 2 to the right $(\bmod 4) . F$ is a surface of genus 5 with $\partial F$ consisting of eight circles, each projecting 2 to 1 onto $\beta$ in $T_{0}$. 
Both $D_{x}$ and $D_{y}^{4}$ lift to homeomorphisms of $F . D_{x}$ lifts to $\widetilde{D}_{x}$ which can be viewed as $1 / 4$ "fractional" Dehn twists about the $\left\{\tilde{x}_{i}\right\}$. In particular $\widetilde{D}_{x}$ fixes pointwise rows 1 and 3 while shifting rows 2 and 4 each three squares to the right $(\bmod 4) . D_{y}^{4}$ lifts to $\widetilde{D}_{y}$ which consists of performing simultaneous Dehn twists about the $\left\{\tilde{y}_{i}\right\}$.

Since both $D_{x}$ and $D_{y}^{4}$ lift to $F, h$ lifts to a homeomorphism $\tilde{h}: F \rightarrow F$. It is easy to see that $\tilde{h}$ fixes pointwise $\partial F$ and that $\tilde{h}$ is homology reducible since $\tilde{h}_{*}$ fixes the nonboundary class $[\gamma]+[\delta]$ in $H_{1}(F)$.

Let $\widetilde{M}=F \times I / \tilde{h}$. All Dehn fillings on $\widetilde{M}$ have positive first Betti number. Moreover, since $\tilde{h}$ fixes pointwise $\partial F$, it follows that the loops $\alpha_{h}, \beta^{2}$ in $\partial M_{h}$ lift to loops $\tilde{\alpha}_{i}, \tilde{\beta}_{i}$ in the eight components of $\partial \widetilde{M}$. Denote by $\widetilde{M}(\mu, \lambda)$ the manifold obtained by Dehn filling on $\widetilde{M}$ with respect to the curves $\tilde{\alpha}_{i}^{\mu} \tilde{\beta}_{i}^{\lambda}$. Then the sequence of covers

$$
\widetilde{M}\left(\mu, \frac{\lambda-\mu}{2}\right) \rightarrow M_{h}(\mu, \lambda-\mu) \rightarrow M(3 \mu, \lambda)
$$

gives the desired cover of $M(3 \mu, \lambda), \mu, \lambda$ odd.

6. In this section we deal with the manifolds $M(2 \mu, \lambda)$. Throughout $\S 6$, we fix $h=\left(R \circ D_{y}^{-3}\right)$ where $R$ is the homeomorphism of $T_{0}$ induced by a $90^{\circ}$ counter-clockwise rotation of the square in Figure 2.

Let $M_{h}=T_{0} \times I / h$. The loop $\alpha_{h}$ is represented in $T_{0} \times I$ by the image of the curve $b \times I$ under a $90^{\circ}$ clockwise rotation of $\partial T_{0} \times\{1\}$.

LEMMA 6.1. $M_{h}$ is bundle equivalent to $M$, with the pair $\left(\alpha_{h}, \beta\right)$ mapping to $(\alpha, \beta)$.

Proof. Let $R^{\prime}$ denote $R$ composed with a $90^{\circ}$ clockwise rotation of $\partial T_{0}$. Then $R^{\prime}$ fixes $\partial T_{0}$ and induces on $\pi_{1}\left(T_{0}, b\right)$ the isomorphism $R_{\#}^{\prime}(x)=x y x^{-1}, R_{\#}^{\prime}(y)=x^{-1}$. A calculation shows that, for any $z \in \pi_{1}\left(T_{0}, b\right)$,

$$
g_{\#}(z)=\left(D_{x}^{-1} \circ R^{\prime} \circ D_{y}^{-3} \circ D_{x}\right)_{\#}(z) .
$$

Thus the isotopy $H$ from $g$ to $D_{x}^{-1} \circ h \circ D_{x}$ rotates $\partial T_{0}$ only $90^{\circ}$ counter-clockwise and hence the bundle isomorphism $H \circ$ $\left(D_{x}^{-1} \times \mathrm{Id}\right): M_{h} \rightarrow M$ sends $\left(\alpha_{h}, \beta\right)$ to $(\alpha, \beta)$.

Now consider $M^{\prime}=T_{0} \times I / h^{4}$, the 4-fold cyclic cover of $M_{h}$. Note that $h^{4}$ fixes $\partial T_{0}$, so we define $\left(\alpha^{\prime}, \beta\right)$ for $M^{\prime}$, where $\alpha^{\prime}=b \times I / h^{4}$. 
LEMMA 6.2. $M^{\prime} \rightarrow M$ is a 4-fold cover, sending the pair of loops $\left(\alpha^{\prime}, \beta\right)$ to the pair $\left(\alpha^{4} \beta, \beta\right)$.

Proof. Note that the lift of $\alpha_{h}^{4}$ to $M^{\prime}$ winds once around $\partial T_{0}$ in the clockwise direction and hence is represented by $\alpha^{\prime} \beta^{-1}$. Thus $\alpha^{\prime}$ projects to $\alpha_{h}^{4} \beta$ in $M_{h}$ which maps to $\alpha^{4} \beta$ in $M$ by Lemma 6.1.

The following is an immediate consequence of Lemma 6.2 and will be used in $\S 6.1$ :

CoRollary 6.3. $M^{\prime}(\mu, \lambda) \rightarrow M(4 \mu, \mu+\lambda) \cong M(4 \mu,-\mu-\lambda)$ is $a$ 4-fold cover.

6.1. Now we prove the following (see also [KL]):

THEOREM B. $M(4 \mu, \lambda)$ has a virtually $\mathbb{Z}$-representable fundamental group.

We begin by considering the 9 -fold cover $S \rightarrow T_{0}$ corresponding to the kernel of the map $\theta: \pi_{1}\left(T_{0}\right) \rightarrow \mathbb{Z} / 3 \oplus \mathbb{Z} / 3$ defined by $\theta([x])=$ $(1,0)$ and $\theta([y])=(0,1)$. Note that both $D_{y}^{-3}$ and $R$ lift to $S$.

Next we construct, for each $d \geq 3$, a cover $F_{d} \rightarrow T_{0}$ as follows: Let $S_{1}, \ldots, S_{d}$ be copies of $S$, each with eight cuts $\left\{\tau_{i}\right\}$ as pictured in Figure 4. Glue the left edge of $\tau_{1}$ in $S_{i}$ to the right edge of $\tau_{1}$ in $S_{i+1}(\bmod d)$. Next glue the left edge of $\tau_{2}$ in $S_{i}$ to the right edge of $\tau_{2}$ in $S_{i-2}(\bmod d)$. Now glue the edges $\tau_{3}, \ldots, \tau_{8}$ so that the gluing is compatible with that of $\tau_{1}, \tau_{2}$ under a simultaneous counter-clockwise rotation by $90^{\circ}$ of each $S_{i}$. Note that the gluing of $\tau_{1}$ determines the pattern for $\tau_{3}, \tau_{5}, \tau_{7}$ while the gluing of $\tau_{2}$ determines that of the $\tau_{4}, \tau_{6}, \tau_{8}$. The surface $F_{3}$, with identifications for $\tau_{i}$ numbered, is pictured in Figure 5. Some of the properties of the surface $F_{d}$ are given in:

LEMMA 6.4. The surface $F_{d}$ is a 9d-fold cover of $T_{0}$. Each component $\tilde{\beta}_{i}$ of $\partial F_{d}$ projects $r_{i}$ to 1 onto $\beta=\partial T_{0}$ for $r_{i} \mid d$.

Now the loop $x$ (resp. $y$ ) in $T_{0}$ is covered by $3 d$ loops $\tilde{x}_{1}, \ldots$, $\tilde{x}_{3 d}$ (resp. $3 d$ loops $\tilde{y}_{1}, \ldots, \tilde{y}_{3 d}$ ) in $F_{d}$ that project 3 to 1 onto $x$ (resp. 3 to 1 onto $y$ ). Thus $D_{y}^{-3}$ lifts to $\widetilde{D}_{y}^{-1}$ consisting of simultaneous negative Dehn twists about the $\left\{\tilde{y}_{i}\right\}$. It follows from the construction of $F_{d}$ that $R$ lifts to $\widetilde{R}$, a simultaneous counter-clockwise rotation by $90^{\circ}$ of each of the $S_{1} \ldots, S_{d}$ in $F_{d}$. Thus $h\left(=R \circ D_{y}^{-3}\right)$ and $h^{4}$ lift to $\tilde{h}$ and $\tilde{h}^{4}$ on $F_{d}$. Note that $\tilde{h}^{4}$ fixes pointwise $\partial F_{d}$. 


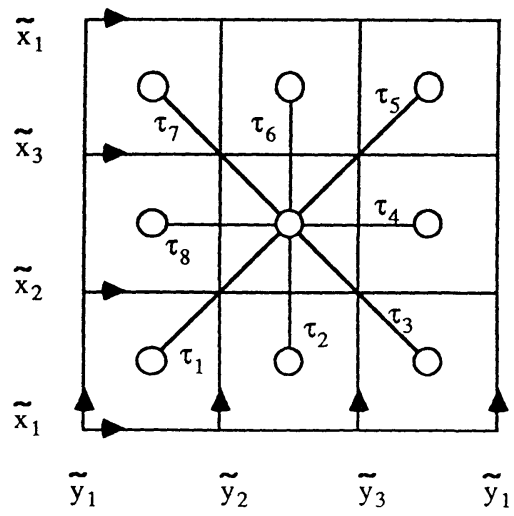

FIGURE 4



$S_{1}$

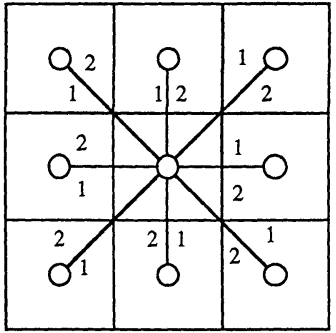

$\mathrm{S}_{2}$

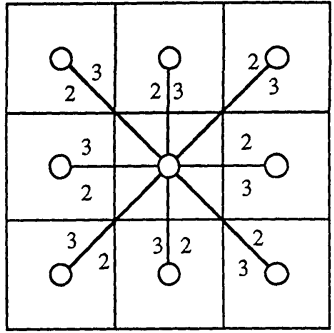

$S_{3}$

FIGURE 5

LemMa 6.5. $\tilde{h}^{4}: F_{d} \rightarrow F_{d}$ is homology reducible.

Proof. A portion of $F_{d}$ is pictured in Figure 6. The non-boundary class $[\gamma]+[\delta]$ in $H_{1}\left(F_{d}\right)$ corresponding to the loops $\gamma, \delta$ is fixed by $\tilde{h}_{*}^{4}$. Indeed, $\widetilde{R}^{4}=\mathrm{Id}$ and $[\gamma]+[\delta]$ is fixed by $\left(\widetilde{D}_{y}^{-1}\right)_{*}$ since $\gamma$ and $\delta$ each intersect the same Dehn twist curves in $\left\{\tilde{y}_{i}\right\}$ with opposite orientations.

Let $\widetilde{M}_{d}=F_{d} \times I / \tilde{h}^{4}$. Now $\widetilde{M}_{d}$ is, by construction, a $9 d$-fold cover of $M^{\prime}$, the 4-fold cyclic cover of $M_{h}$, hence $\widetilde{M}_{d} \rightarrow M$ is a $36 d$-fold covering space (see Lemma 6.2). Furthermore, Lemma 6.5 implies that any Dehn filling on $\widetilde{M}_{d}$ yields a manifold with positive first Betti number.

We complete the proof of Theorem B by constructing, for each $(4 \mu, \lambda)$ coprime, a cover $N \rightarrow M(4 \mu, \lambda), \beta_{1}(N) \geq 1$, gotten by Dehn filling on an appropriate $\widetilde{M}_{d}$. Since $M(0, \pm 1)$ itself has positive first Betti number, we exclude this case. 


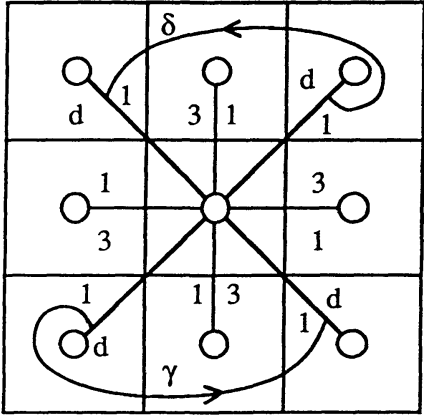

$S_{1}$

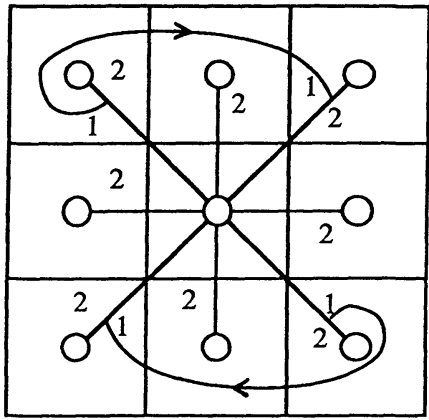

$\mathrm{S}_{2}$

FIGURE 6

Recall that $M^{\prime}(\mu, \lambda-\mu) \rightarrow M(4 \mu, \lambda) \cong M(4 \mu,-\lambda)$ is a 4-fold cover by Corollary 6.3. Since $(4 \mu, \lambda) \neq(0, \pm 1)$, by changing the sign of $\lambda$ if necessary, we can assume that either $\lambda=\mu= \pm 1$ or $|\lambda-\mu| \geq 3$. In the first case the loop $\alpha^{\prime}$ in $\partial M^{\prime}$ lifts to loops $\left\{c_{i}\right\}$ in $\partial \widetilde{M}_{d}$ for any $d$. In the second case the loop $\left(\alpha^{\prime}\right)^{\mu} \beta^{\lambda-\mu}$ in $\partial \widetilde{M}$ lifts to loops $\left\{c_{i}\right\}$ in $\partial \widetilde{M}_{d}$ for $d=|\lambda-\mu|$. In both cases we obtain $N \rightarrow M(4 \mu, \lambda)$ by Dehn filling on $\widetilde{M}_{d}$ with respect to the loops $\left\{c_{i}\right\}$ in $\partial \widetilde{M}_{d}$. This completes the proof of Theorem B.

As an example, consider the case $M(8,-1) \cong M(8,1)$. Then $M^{\prime}(2,-3) \rightarrow M(8,1)$ and the $(2,-3)$ loop in $M^{\prime}$ lifts to loops $\left\{c_{i}\right\}$ in the boundary components of $\widetilde{M}_{3} . N$ is gotten by Dehn filling on $\widetilde{M}_{3}$ with respect to the loops $\left\{c_{i}\right\}$ (see Figure 5).

6.2. Proposition C. $M(2 \mu, \lambda)$ has a virtually $\mathbb{Z}$-representable fundamental group if $\lambda \equiv \pm 7 \mu(\bmod 15)$.

Consider the 9-fold cover $S \rightarrow T_{0}$ described in $\S 6.1$, and construct a new cover $F \rightarrow T_{0}$ by making eight cuts in $S$ and identifying the edges as shown in Figure 7. The surface $F$ has genus 4 and $\partial F$ consists of 3 circles: $\tilde{\beta}_{1}$ that projects 5-1 onto $\beta, \tilde{\beta}_{2}$ projecting 3-1 onto $\beta$, and $\tilde{\beta}_{3}$ projecting $1-1$ onto $\beta$. The loop $x$ (resp. $y$ ) in $T_{0}$ is covered by the three loops $\tilde{x}_{1}, \tilde{x}_{2}, \tilde{x}_{3}$ (resp. $\tilde{y}_{1}, \tilde{y}_{2}, \tilde{y}_{3}$ ) which project 3-1 onto $x$ (resp. onto $y$ ).

It follows from the construction of $F$ that $R$ lifts to $\widetilde{R}$ the homeomorphism induced by a $90^{\circ}$ counter-clockwise rotation, and that $D_{y}^{-3}$ lifts to $\widetilde{D}_{y}^{-1}$ given by simultaneous negative Dehn twists about the $\left\{\tilde{y}_{i}\right\}$. Hence $h\left(=R \circ D_{y}^{-3}\right)$ lifts to $\tilde{h}$. 


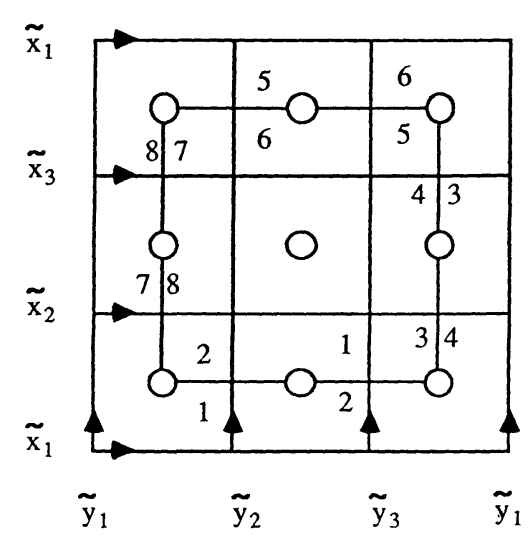

FIGURE 7

LEMMA 6.6. $\tilde{h}^{2}$ is homology reducible.

Proof. $\tilde{h}_{*}^{2}$ fixes the non-boundary class $\left[\tilde{x}_{2}\right]-\left[\tilde{x}_{3}\right]$ in $H_{1}(F)$ (see Figure 7).

Let $\widetilde{M}=F \times I / \tilde{h}^{2}$. Then $\widetilde{M}$ is an 18 -fold cover of $M_{h}$. Since $\tilde{h}^{2}$ rotates each component $\tilde{\beta}_{i}$ of $\partial F$ one half turn counter-clockwise, we can choose on each component $T_{i} \subset \partial \widetilde{M}$ loops $\left(\tilde{\alpha}_{i}, \tilde{\beta}_{i}\right)$ where $\left(\tilde{\alpha}_{1}, \tilde{\beta}_{1}\right)$ projects to $\left(\alpha_{h}^{2} \beta^{-2}, \beta^{5}\right),\left(\tilde{\alpha}_{2}, \tilde{\beta}_{2}\right)$ projects to $\left(\alpha_{h}^{2} \beta^{-1}, \beta^{3}\right)$ and $\left(\tilde{\alpha}_{3}, \tilde{\beta}_{3}\right)$ projects to $\left(\alpha_{h}^{2}, \beta\right)$ in $M_{h}$.

Now our proposition follows, since by the above paragraph any loop in $\partial M_{h}$ of the form $\alpha_{h}^{2 \mu} \beta^{\lambda}, \lambda \equiv-7 \mu(\bmod 15)$, lifts to loops $\left\{c_{i}\right\}$ in each component $T_{i}$ of $\partial \widetilde{M}$. Dehn filling on $\widetilde{M}$ with respect to the loops $\left\{c_{i}\right\}$ provides a cover $N \rightarrow M_{h}(2 \mu, \lambda) \cong M(2 \mu, \lambda)$, the last isomorphism by Lemma 6.1 .

6.3. Remark. By similar arguments, we can show that $M(2 \mu, \lambda)$ has a virtually $\mathbb{Z}$-representable fundamental group if $\lambda \equiv \pm 3 \mu$ $(\bmod 7)$. These cases have been done in [H1] and [N] by different methods (see $\S 3$ ). Consider the cover $F \rightarrow T_{0}$ in Figure 8, obtained from 3 copies of $S$ by removing the interiors of the four shaded regions in each copy of $S$ and identifying the edges as numbered. The reader should check the following: $\partial F$ consists of 3 circles, each pro= jecting 7 to 1 onto $\beta=\partial T_{0} ; h^{2}$ lifts to a homology reducible map $\tilde{h}^{2}: F \rightarrow F$; and the loop $\alpha^{2 \mu} \beta^{\lambda}, \lambda \equiv \pm 3 \mu(\bmod 7)$, in $M$ lifts to loops in $\widetilde{M}=F \times I / \tilde{h}^{2}$.

7. Singular boundary curve systems for $M$. In this section we study singular incompressible surfaces in $M$. Given a cover $N \rightarrow M(\mu, \lambda)$ 




$s_{1}$

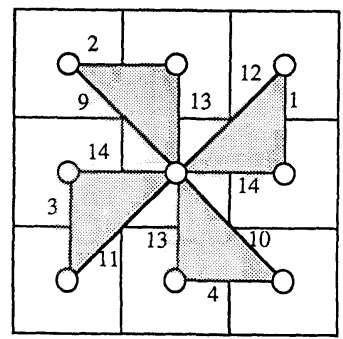

$S_{2}$

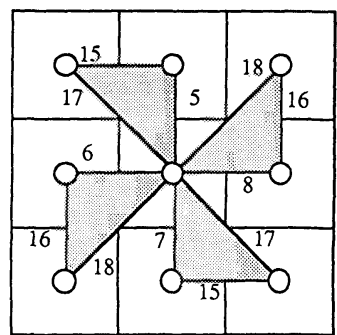

$\mathrm{S}_{3}$

FIGURE 8

obtained by Dehn filling on $\widetilde{M} \rightarrow M$, then $\beta_{1}(N) \geq \beta_{1}(\widetilde{M})-\beta_{0}(\partial \widetilde{M})$. Hempel shows ([H2]) that this inequality is strict if and only if there is an incompressible, boundary incompressible surface $F$ in $\widetilde{M}$ such that $\partial F$ consists of a non-empty collection of Dehn filling curves. This surface $F$ projects to a singular surface in $M$ whose boundary curves are $\alpha^{\mu} \beta^{\lambda}$, and we say that $\left\{\alpha^{\mu} \beta^{\lambda}\right\}$ is a singular boundary curve system for $M$.

In [H2] the curves $\left\{\alpha^{3}\right\},\{\alpha, \beta\}$, and $\left\{\alpha \beta^{-1}\right\}$ are shown to be singular boundary curve systems. We show:

THEOREM D. The curves $\left\{\alpha^{2} \beta\right\},\left\{\alpha^{2} \beta^{-1}\right\},\left\{\alpha^{3} \beta\right\}$ and $\left\{\alpha^{3} \beta^{-1}\right\}$ are singular boundary curve systems for $M$.

(a) The curves $\alpha^{3} \beta^{ \pm 1}$ : We use the 3-fold cover $M_{h} \rightarrow M$ for $h=$ $D_{x}^{2} \circ D_{y}^{-4} \circ D_{x} \circ D_{y}^{-4} \circ D_{x}$ described in $\S 5$.

By Lemma 5.1, $M_{h}(1,0) \rightarrow M(3,1)$ is a 3-fold covering. Now consider the 8-fold cover $F \rightarrow T_{0}$, pictured in Figure 9, to which $h$ lifts (see $\S 5$ ): Denote this lift by $\tilde{h}$. Note that $\tilde{h}$ fixes pointwise the eight components of $\partial F$ and that $\tilde{h}$ is not homology reducible.

Let $\widetilde{M}=F \times I / \tilde{h}$. By construction, the loop $\alpha_{h}$ in $M_{h}$ lifts to eight loops $\tilde{\alpha}_{1}, \ldots, \tilde{\alpha}_{8}$ in $\partial \widetilde{M}$-indexed so that the loops $\left(\tilde{\alpha}_{i}, \tilde{\beta}_{i}\right)$ lie in the $i$ th boundary torus of $\widetilde{M}$. Thus the loops $\tilde{\alpha}_{i}$ project to $\alpha^{3} \beta$ in $\partial M$ and Dehn filling on $\widetilde{M}$ with respect to the $\left\{\tilde{\alpha}_{i}\right\}$ gives a cover $N \rightarrow M(3,1) \cong M(3,-1)$.

LEMMA 7.1. There exist relations among $\left\{\left[\tilde{\alpha}_{i}\right]\right\}$ in $H_{1}(\widetilde{M})$; hence $\beta_{1}(N)>\beta_{1}(\widetilde{M})-\beta_{0}(\widetilde{M})$.

Proof. We have $\left[\tilde{\alpha}_{2}\right]-\left[\tilde{\alpha}_{1}\right]=\left[\tilde{\alpha}_{6}\right]-\left[\tilde{\alpha}_{5}\right]$ in $H_{1}(\widetilde{M})$. One computes $\left[\tilde{\alpha}_{j}\right]-\left[\tilde{\alpha}_{i}\right]$ as follows. Let $\sigma_{i j}$ be a simple path in $F \times\{0\}$ from 


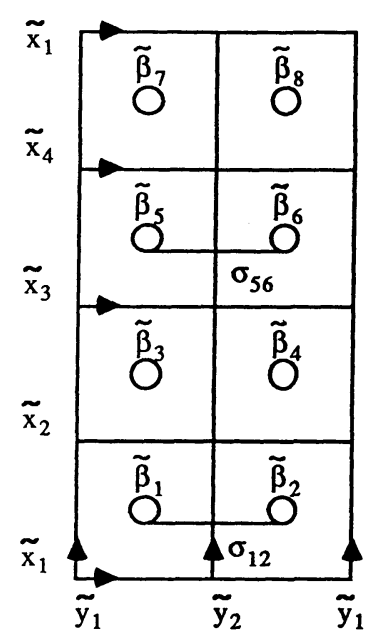

FIGURE 9

$\tilde{\alpha}_{i} \cap F$ to $\tilde{\alpha}_{j} \cap F$. Then the disk $\sigma_{i j} \times I \subset F \times I$ provides the relation $\left[\tilde{\alpha}_{j}\right]-\left[\tilde{\alpha}_{i}\right]=\left[\tilde{h}\left(\sigma_{i j}\right) * \sigma_{i j}^{-1}\right]$ where $*$ denotes path composition.

Now $\sigma_{12}$ and $\sigma_{56}$ can be chosen as in Figure 9, and $\left[\tilde{h}\left(\sigma_{12}\right) * \sigma_{12}^{-1}\right]=$ $\left[\tilde{h}\left(\sigma_{56}\right) * \sigma_{56}^{-1}\right]$ in $H_{1}(\widetilde{M})$ since $\widetilde{D}_{x}$ fixes $\sigma_{12}$ and $\sigma_{56}$ pointwise and they both intersect the Dehn twist curve $\tilde{y}_{2}$.

(b) The curves $\alpha^{2} \beta^{ \pm 1}$ : Consider the bundle $M_{f}=T_{0} \times I / f$ for $f=\left(D_{x}^{-1} \circ D_{y}^{5}\right)^{2}$.

LemMa 7.2. $M_{f}$ is a 2-fold cover of $M$. The pair $\left(\alpha_{f}, \beta\right)$ maps to the pair $\left(\alpha^{2} \beta^{-1}, \beta\right)$.

Proof. Let $g^{\prime}=\left(D_{x}^{-1} \circ D_{y}^{2} \circ D_{x}^{-1}\right) \circ g^{2} \circ\left(D_{x}^{-1} \circ D_{y}^{2} \circ D_{x}^{-1}\right)^{-1}$. We have, for any $z \in \pi_{1}\left(T_{0}, b\right)$,

$$
g_{\#}^{\prime}(z)=\left(x y x^{-1} y^{-1}\right)^{-1} f_{\#}(z)\left(x y x^{-1} y^{-1}\right) .
$$

Thus the isotopy $H$ between $g^{\prime}$ and $f$ rotates $\partial T$ one full turn clockwise, so that the bundle isomorphism $\left\{\left(D_{x}^{-1} \circ D_{y}^{2} \circ D_{x}^{-1}\right)^{-1} \times\right.$ Id $\} \circ$ $H: M_{f} \rightarrow M_{g^{2}}$ sends the pair $\left(\alpha_{f}, \beta\right)$ to $\left(\alpha_{g^{2}} \beta^{-1}, \beta\right)$ which projectș to $\left(\alpha^{2} \beta^{-1}, \beta\right)$ in $M$.

By Lemma 7.2 $M_{f}(1,0) \rightarrow M(2,-1)$ is a 2 -fold cover. Now consider the 10-fold cover $F \rightarrow T_{0}$, pictured in Figure 10 to which $f$ 




FIGURE 10

lifts. Denote the lift of $f$ by $\tilde{f}$ and let $\widetilde{M}=F \times I / \tilde{f}$. Now $\tilde{f}$ fixes pointwise the eight boundary circles of $F$. Denote by $\tilde{\alpha}_{i}$ a lift of $\alpha_{f}$ to $\partial \widetilde{M}$, indexed so that the loops $\left(\tilde{\alpha}_{i}, \tilde{\beta}_{i}\right)$ lie on the $i$ th boundary torus of $\widetilde{M}$. Thus the loops $\tilde{\alpha}_{i}$ project to $\alpha^{2} \beta^{-1}$ in $M$ and Dehn filling on $\widetilde{M}$ with respect to $\left\{\tilde{\alpha}_{i}\right\}$ gives a cover $N \rightarrow M(2,-1) \cong$ $M(2,1)$.

LEMMA 7.3. There exist relations among $\left\{\left[\tilde{\alpha}_{i}\right]\right\}$ in $h_{1}(\widetilde{M})$; hence $\beta_{1}(N)>\beta(\widetilde{M})-\beta_{0}(\widetilde{M})$.

Proof. We have $\left[\tilde{\alpha}_{2}\right]-\left[\tilde{\alpha}_{1}\right]=\left[\tilde{\alpha}_{6}\right]-\left[\tilde{\alpha}_{5}\right]$ in $H_{1}(\widetilde{M})$ by an argument identical to that in Lemma 7.1.

\section{REFERENCES}

[B] M. Baker, Covers of Dehn fillings on once-punctured torus bundles II, Proc. Amer. Math. Soc., 110 (1990), 1099-1108.

[H1] J. Hempel, Coverings of Dehn fillings of surface bundles, Topology Appl., 24 (1986), 157-170.

[H2] _ Coverings of Dehn fillings of surface bundles II, Topology Appl., 26 (1987), 163-173.

[KL] S. Kojima and D. Long, Virtual Betti numbers of some hyperbolic 3-manifolds, preprint. 
[N] A. Nicas, An infinite family of hyperbolic non-Haken 3-manifolds with vanishing Whitehead groups, Math. Proc. Camb. Phil. Soc., 99 (1986), 239-246.

[T] W. Thurston, The geometry and topology of 3-manifolds, Notes, Princeton University, 1977.

Received February 26, 1990.

\section{IHES}

91440 BURES-SUR-YVETTE

FRANCE

AND

UNIVERSITÉ DE RENNES I

35042 RENNES CEDEX

FRANCE 


\section{PACIFIC JOURNAL OF MATHEMATICS EDITORS}

\author{
V. S. VARADARAJAN \\ (Managing Editor) \\ University of California \\ Los Angeles, CA 90024-1555-05 \\ Herbert Clemens \\ University of Utah \\ Salt Lake City, UT 84112 \\ THOMAS ENRIGHT \\ University of California, San Diego \\ La Jolla, CA 92093
}

R. FINN

Stanford University

Stanford, CA 94305

HeRmann FlaschKa

University of Arizona

Tucson, AZ 85721

VAUGHAN F. R. JoNES

University of California

Berkeley, CA 94720

SteVen KeRCKHOFF

Stanford University

Stanford, CA 94305
C. C. MOORE

University of California

Berkeley, CA 94720

MaRTin ScharLemanN

University of California

Santa Barbara, CA 93106

HAROLd STARK

University of California, San Diego

La Jolla, CA 92093

\section{ASSOCIATE EDITORS \\ R. ARENS \\ E. F. BECKENBACH (1906-1982) \\ B. H. NeumanN \\ F. WoLf \\ (1904-1989) \\ K. YoshidA \\ SUPPORTING INSTITUTIONS \\ UNIVERSITY OF ARIZONA \\ UNIVERSITY OF BRITISH COLUMBIA \\ CALIFORNIA INSTITUTE OF TECHNOLOGY \\ UNIVERSITY OF CALIFORNIA \\ MONTANA STATE UNIVERSITY \\ UNIVERSITY OF NEVADA, RENO \\ NEW MEXICO STATE UNIVERSITY OREGON STATE UNIVERSITY \\ UNIVERSITY OF OREGON \\ UNIVERSITY OF SOUTHERN CALIFORNIA \\ STANFORD UNIVERSITY \\ UNIVERSITY OF HAWAII \\ UNIVERSITY OF TOKYO \\ UNIVERSITY OF UTAH \\ WASHINGTON STATE UNIVERSITY UNIVERSITY OF WASHINGTON}

The Supporting Institutions listed above contribute to the cost of publication of this Journal, but they are not owners or publishers and have no responsibility for its content or policies.

Mathematical papers intended for publication in the Pacific Journal of Mathematics should be in typed form or offset-reproduced (not dittoed), double spaced with large margins. Please do not use built up fractions in the text of the manuscript. However, you may use them in the displayed equations. Underline Greek letters in red, German in green, and script in blue. The first paragraph must be capable of being used separately as a synopsis of the entire paper. In particular it should contain no bibliographic references. Please propose a heading for the odd numbered pages of less than 35 characters. Manuscripts, in triplicate, may be sent to any one of the editors. Please classify according to the 1991 Mathematics Subject Classification scheme which can be found in the December index volumes of Mathematical Reviews. Supply name and address of author to whom proofs should be sent. All other communications should be addressed to the managing editor, or Elaine Barth, University of California, Los Angeles, California 90024-1555-05.

There are page-charges associated with articles appearing in the Pacific Journal of Mathematics. These charges are expected to be paid by the author's University, Government Agency or Company. If the author or authors do not have access to such Institutional support these charges are waived. Single authors will receive 50 free reprints; joint authors will receive a total of 100 free reprints. Additional copies may be obtained at cost in multiples of 50 .

The Pacific Journal of Mathematics (ISSN 0030-8730) is published monthly except for July and August. Regular subscription rate: $\$ 190.00$ a year (10 issues). Special rate: $\$ 95.00$ a year to individual members of supporting institutions.

Subscriptions, orders for numbers issued in the last three calendar years, and changes of address should be sent to Pacific Journal of Mathematics, P.O. Box 969, Carmel Valley, CA 93924, U.S.A. Old back numbers obtainable from Kraus Periodicals Co., Route 100, Millwood, NY 10546.

The Pacific Journal of Mathematics at P.O. Box 969, Carmel Valley, CA 93924 (ISSN 0030-8730) is published monthly except for July and August. Second-class postage paid at Carmel Valley, California 93924, and additional mailing offices. Postmaster: send address changes to Pacific Journal of Mathematics, P.O. Box 969, Carmel Valley, CA 93924.

PUBLISHED BY PACIFIC JOURNAL OF MATHEMATICS, A NON-PROFIT CORPORATION Copyright (C) 1991 by Pacific Journal of Mathematics 


\section{Pacific Journal of Mathematics}

Vol. 150, No. $2 \quad$ October, 1991

Selman Akbulut and Henry Churchill King, Rational structures on

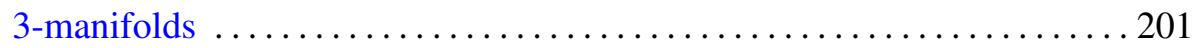

Mark Baker, On coverings of figure eight knot surgeries $\ldots \ldots \ldots \ldots \ldots 215$

Christopher Michael Brislawn, Traceable integral kernels on countably generated measure spaces . ...........................229

William Chin, Crossed products and generalized inner actions of Hopf algebras

Tadeusz Figiel, William Buhmann Johnson and Gideon Schechtman, Factorizations of natural embeddings of $l_{p}^{n}$ into $L_{r}$. II $\ldots \ldots \ldots \ldots \ldots 261$

David Howard Gluck, Character value estimates for groups of Lie type ... 279

Charn-Huen Kan, Norming vectors of linear operators between $L_{p}$

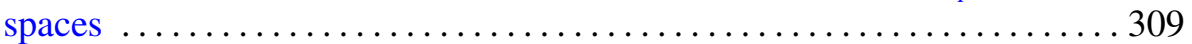

Marko Kranjc, Embedding a 2-complex $K$ in $\mathbb{R}^{4}$ when $H^{2}(K)$ is a cyclic

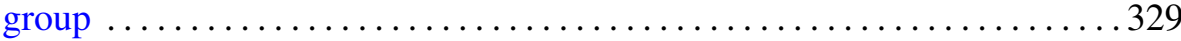

Ka-Lam Kueh, The remainder terms aspect of the theory of the Riemann

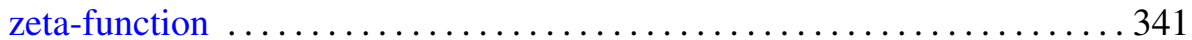

J. A. Marti, Sur la rigidité comparée de fonctions, distributions, ou hyperfonctions analytiques par rapport à un groupe de variables

Margherita Roggero and Paolo Valabrega, Chern classes and cohomology for rank 2 reflexive sheaves on $\mathbf{P}^{3}$ 